Itinéraires Itinéraires

Littérature, textes, cultures

\title{
L'éthos de l'adolescent dans les mondes numériques : le rôle des destinataires
}

Teenager's Ethos in the Digital Worlds: The Role of Recipients

Jocelyn Lachance

\section{CpenEdition}

\section{Journals}

Édition électronique

URL : http://journals.openedition.org/itineraires/3156

DOI : $10.4000 /$ itineraires.3156

ISSN : 2427-920X

Éditeur

Pléiade

Référence électronique

Jocelyn Lachance, «L'éthos de l'adolescent dans les mondes numériques : le rôle des destinataires », Itinéraires [En ligne], 2015-3 | 2016, mis en ligne le 01 juillet 2016, consulté le 19 avril 2019. URL:

http://journals.openedition.org/itineraires/3156; DOI : 10.4000/itineraires.3156

Ce document a été généré automatiquement le 19 avril 2019

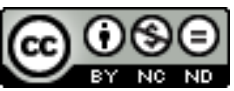

Itinéraires est mis à disposition selon les termes de la licence Creative Commons Attribution - Pas d'Utilisation Commerciale - Pas de Modification 4.0 International. 


\title{
L'éthos de l'adolescent dans les mondes numériques : le rôle des destinataires
}

\author{
Teenager's Ethos in the Digital Worlds: The Role of Recipients
}

\author{
Jocelyn Lachance
}

1 Avec l'effervescence des pratiques numériques, leur diversification et leur renouvellement, il devient hasardeux de définir quels sont les usages spécifiques des technologies de l'image et de la communication des adolescents. En effet, si le sens commun attribue aux plus jeunes de nos sociétés la tentation de la mise en scène sur Internet, nous savons que l'exposition de soi ne concerne pas seulement les adolescents. La mise en scène de la parentalité sur les réseaux sociaux en est un bon exemple: des parents vont mettre en ligne des photos et des vidéos de leurs enfants, de leur famille, en y projetant une image délibérément positive. Dans ce contexte où se côtoient désormais sur la toile enfants, adolescents, adultes et retraités, en quoi les ados se distinguent-ils de leurs cadets et de leurs aînés?

2 À l'ère du numérique, l'auditoire est immense, sans limite seraient tentés de dire certains. Pourtant, ce vaste territoire se caractérise par une multiplication des cloisons et des corridors que seuls des privilégiés peuvent emprunter. Ainsi, il est possible d'accéder à sa boîte mail, à son compte en banque ; possible de s'inscrire sur des forums de discussion, de partager en ligne des photos sur Facebook ou via Snapchat, de publier une vidéo sur YouTube ou d'échanger avec Younow' ${ }^{1}$. Il est tout aussi facile d'envoyer un message à un ami proche, mais lointain, sur le moment. Ces quelques exemples nous rappellent un fait banal, mais parfois oublié: dans les mondes numériques, les interlocuteurs sont nombreux, diversifiés, mais surtout, ils ne sont jamais les mêmes, ce qui complexifie passablement l'analyse du sens véritable que prennent les paroles, les mots, les photos et les vidéos qui y circulent. Cette complexité s'amplifie lorsque le destinataire imaginé par le diffuseur ne correspond pas exactement aux interlocuteurs réels. C'est le cas lorsqu'un individu s'étonne d'apprendre que telle ou telle personne a eu accès à du contenu diffusé sur la Toile, sans qu'il ait été visé par l'envoi. 
$3 \mathrm{Au}$ cours de cet article, nous proposerons une lecture socio-anthropologique (Lachance 2012, Goguel d'Allondans et Lachance 2014, Jeffrey et al. 2016) des mises en scène de l'adolescent dans les mondes numériques, des mises en scènes comprises ici comme des performances fortement imprégnées par le travail identitaire caractéristique de cette période de la vie. Dans la lignée de l'anthropologie culturelle de Christoph Wulf, nous comprenons ici les performances comme des configurations symboliques où le corps tient la première place au cours d'interactions situées (Wulf 2007). Ces performances seraient donc constitutives d'un éthos numérique, au sens où l'entend Erwing Goffman (1973). Nous verrons que ces performances sont plurielles puisque fortement liées aux destinataires diversifiés et visés par la diffusion de mises en scène contextualisées. Ainsi, nous proposons d'explorer le rôle du destinataire dans le déploiement de ces performances elles-mêmes constitutives d'un éthos numérique spécifique. À la diversité des regards recherchés sur Internet et posés sur soi via les technologies de l'image et de la communication correspondrait alors un éventail de postures répondant à différentes conventions, selon la relation qui s'instaure avec l'interlocuteur recherché. À l'instar de leurs aînés, les adolescents vont principalement s'adresser à quatre différents cercles d'interlocuteurs : soi-même (ex. : visionnage sur un écran d'une photo ou d'une vidéo prise par soi-même), une personne unique et désignée (ex.: Snapchat), un groupe restreint dont l'on connaît l'identité des membres (ex. : Facebook), un groupe illimité dont l'on ne connaît pas l'identité des membres (ex.: YouTube). Dans le contexte de l'adolescence, l'interpellation de ces regards multiples implique la constitution d'un éthos numérique révélateur de la nécessité anthropologique de s'autonomiser, d'où l'importance de l'appropriation progressive de codes de présentation de soi, la confrontation progressive de son image aux autres et l'élaboration du sentiment de prendre des risques. Après l'exploration de ces quatre cercles d'interlocuteurs, nous conclurons en revenant sur les enjeux identitaires que cet éthos semble révéler.

\section{Se présenter à soi-même : s'engager dans une démarche de découverte de soi}

4 Le premier cercle d'interlocuteur se restreint au producteur lui-même. La photo ou la vidéo est destinée à un usage intime et personnel. En fait, si Internet a rendu possible la diffusion à grande échelle des photos et des vidéos, cela n'empêche pas l'existence d'images destinées à aucun autre public que soi-même. Des photos et des vidéos sont produites dans l'intimité et destinées à être visionnées par son producteur. Cette pratique s'inscrit la plupart du temps dans une volonté avouée de mieux se connaître : il s'agit de s'observer à travers la médiation de l'écran, en attribuant à ce nouveau point de vue sur soi la qualité généralement donnée à un regard extérieur porté sur soi (Lachance 2013a). En d'autres termes, l'image produite et projetée sur l'écran numérique donne subjectivement un aperçu de ce corps tel qu'il serait vu par l'autre. Des adolescents vont alors tenter de prendre une photo d'eux-mêmes, sur le vif, une photo interprétée comme étant authentique, afin de se regarder tel que l'on se présenterait aux autres, sans apparat. Dans d'autres cas, c'est bien entendu la mise en scène que des jeunes vont confronter à leur propre regard. Un éventail de vêtements est utilisé, des postures sont testées, des sourires et des regards joués, des maquillages tentés. D'emblée, une telle performance de l'adolescent à l'ère du numérique annonce sa complexité : parfois, il 
importe d'éradiquer les artifices de la mise en scène. Dans d'autres contextes, l'objectif est au contraire d'apprendre à en maîtriser les codes.

Les producteurs de photos et de vidéos sont animés par le désir de produire une photo ou une vidéo authentique ou, au contraire, mise en scène. Les documents visuels destinés à un usage intime participent de la création d'un repère intermédiaire entre le regard subjectif que porte l'adolescent sur lui-même en s'observant dans le miroir et le regard tout aussi subjectif que l'autre porte sur lui en face-à-face. En d'autres termes, le numérique favorise la production d'un espace dans l'interstice séparant la représentation que l'individu se fait de lui-même de la représentation que l'autre développerait à son endroit. La photo et la vidéo produites dans ce contexte constituent des symboles au premier sens du terme: ils réunissent l'irréconciliable, puisqu'ils représentent ce que l'autre voit de lui-même, à partir d'une position inatteignable que le producteur de documents visuels a l'impression de prendre provisoirement. De tels documents visuels, mettant en scène le corps, peuvent alors être compris comme des territoires où convergent regard de soi sur soi et regard de l'autre sur soi. Des territoires où se joue une renégociation entre ce que l'on imagine de soi et ce que l'on croit voir à travers les yeux de l'autre.

6 Dans ce contexte, il s'agit moins de produire un éthos définitif afin de gagner la confiance d'un autre que d'engager celui-ci afin de gagner de la confiance en soi $^{2}$. Sur un registre plus intime, certains adolescents vont alors photographier ou filmer leur corps nu afin de s'observer «de l'extérieur». Certains documents produits s'appuieront donc sur une logique d'authenticité. Il s'agit de s'observer «au naturel», sans apparat, comme l'individu se croit être au quotidien, au lever, après une nuit de sommeil, etc. Ainsi, la production d'un éthos sur le mode de l'authenticité suppose, d'une part, qu'une photo de soi peut effectivement représenter la personne «en vrai » et, d'autre part, que cette confrontation peut permettre une certaine découverte de soi. Sous le mode de la révélation, la photo et la vidéo produites pour soi donnent alors aux individus des informations insoupçonnées sur eux-mêmes, malgré leur caractère profondément subjectif (Schwarz 2010a, Lachance 2013b).

7 D'autres documents visuels s'appuieront plutôt sur une logique de mise en scène. Si la logique d'authenticité implique un laisser-aller, un abandon du corps sous l'œil de l'objectif, la mise en scène implique une préparation mobilisant un ensemble de connaissances. Connaissances de l'usage d'un appareil numérique certes, mais aussi des codes inhérents à une esthétisation efficace du corps à exposer, puis, au besoin, à modifier avec des logiciels de retouche de photos et de vidéos. En un mot, il s'agit d'expérimenter ces codes, de tester les limites de conventions avec l'objectif ultime de se présenter aux autres. Les logiques d'authenticité et de mise en scène ne sont donc pas dépendantes l'une de l'autre, mais se présentent plutôt dans leur continuité : si les photos et les vidéos «authentiques " révèlent à des adolescents la "vérité » de leur corps, les photos et les vidéos mises en scène sont les preuves tangibles d'une action délibérée et possiblement bénéfique de ces adolescents sur cette " vérité ", d'où le caractère à la fois risqué et rassurant de cette opération.

8 Comme le souligne depuis plusieurs années le socio-anthropologue David Le Breton, le corps est devenu dans le contexte hypermoderne un support de l'identité: en le modelant, il est possible de donner une forme visible à ce que l'on croit être (Le Breton 2008). Mais entre l'identité et le corps, l'image s'impose dans la vie des plus jeunes. Il existe désormais un territoire intermédiaire d'apprivoisement de soi. C'est pourquoi la 
frontière entre ce qui est vrai et ce qui est faux semble de moins en moins claire et surtout de moins en moins significative aux yeux des adolescents. Les photos et les vidéos produites sont des théâtralisations de la " vérité » subjective de soi. Elles rendent visibles une démarche loin d'être nouvelle mais qui, autrefois, se déroulait sans laisser de trace : apprendre à se connaître, se regarder pour se comprendre, afin de s'apprivoiser et de s'ajuster au regard des normes à travers la confrontation de soi à l'autre. Les productions de photos et de vidéos à usage personnel et unique sont ainsi devenues les coulisses principales pour l'acteur qui affronte les peurs de ses premières représentations publiques. Une telle performance se construit d'abord dans la mise en tension entre découverte de soi et présentation de soi aux autres, ce qui entraine généralement chez les adolescents une réflexion sur la démarche à emprunter et parfois même la construction d'un discours pour accompagner les documents présentés.

\section{Se présenter à une personne significative : s'engager et se risquer dans la relation}

9 Le deuxième cercle d'interlocuteurs consiste en une seule personne significative, désignée par le locuteur. Par exemple, l'adolescent envoie un « sexto » à un ami ou à une amie choisis, petit copain ou petite copine, via Snapchat ${ }^{3}$. Il s'adresse exclusivement à cette personne, marquant ainsi l'importance de la relation privilégiée qui les unit (Lenhart 2009, Schwarz 2010b, Lavoie 2013). Le regard de l'autre est interpellé. Dans ce contexte, deux univers de significations se rencontrent parfois. D'abord, comme l'a bien montré le psychanalyste Serge Tisseron (2010), il s'agit d'un désir d'extimité, c'est-à-dire que les personnes mettent en avant une partie de leur intimité afin d'obtenir des rétroactions de la part de personnes significatives. Cette tendance importante et nécessaire participe de la construction identitaire, mais aussi de la sociabilité. Ensuite, l'envoi d'une photo ou d'une vidéo, en particulier lorsqu'elles peuvent être compromettantes à cause de leur caractère sexuel ou transgressif, est souvent vécu comme une forme d'engagement entre les personnes impliquées (Lachance 2013a). Le don d'une photo ou d'une vidéo compromettante souligne la confiance donnée à celle ou celui qui la reçoit. En la gardant pour lui, ce dernier vient prouver la confiance qui lui a été donnée. L'éthos numérique se construit également à partir d'une présentation de soi comprise comme un test, avec sa part de prise de risque. Car, que ce soit comme désir d'extimité ou rite d'engagement, l'usage de la photo et de la vidéo numérique comporte toujours le risque du commentaire, de la diffusion et du détournement.

Le rite d'engagement suppose la mise en gage de l'image de soi. Sans la donation d'une image révélant une part cachée, voire intime de soi, nul ne peut imputer à la photo ou à la vidéo valeur de symbole d'un lien de confiance. En d'autres termes, ce n'est pas seulement parce que la photo ou la vidéo numérique donnée à une personne désignée peut être éventuellement diffusée qu'elle prend sa valeur. C'est aussi et d'abord parce que cette photo ou cette vidéo recèlent pour l'adolescent ou l'adolescente qui la donne une part significative d'intimité. Ainsi, ce document visuel ne peut se limiter à une mise en scène de soi, elle doit aussi comporter une part d'authenticité aux yeux de celui qui la donne. Sans surprise, les photos et les vidéos utilisées dans le contexte de rite d'engagement exposent des adolescents dans des situations « naturelles", sans filtre : des documents les montrant dans l'intimité de leur chambre à coucher ou dans l'effervescence d'un moment festif comptent parmi les exemples les plus connus. 
11 Le passage du premier cercle d'interlocuteur (soi-même) au second (une personne significative) implique donc une dimension relationnelle qui s'ajoute à la dimension identitaire de la production de photos et de vidéos. C'est l'entrée sur la scène de l'adolescence quotidienne se déroulant dans les espaces numériques ${ }^{4}$ : au regard imaginé que l'autre porte sur soi s'impose désormais le regard unique et bien réel d'une personne significative mais choisie. Par conséquent, l'adolescent passe subtilement de la révélation ou de la présentation de soi pour soi, à la révélation ou à la présentation de soi pour un autre imaginé, puis pour un autre réel. L'éthos de l'adolescent dans les espaces numériques se caractérise donc par une multiplication des intermédiaires qui s'accompagne d'une multiplication des interlocuteurs. L'une des caractéristiques de l'adolescence hypermoderne (Lachance 2011b) consiste dans cette aisance à produire de nouveaux espaces d'expérimentation de soi, tout en jouant avec les possibilités inhérentes aux regards nombreux qu'ils peuvent attirer. Dans le même temps, une nouvelle forme d'inégalité surgit, départageant ceux qui se jouent de la multiplication des possibilités de ceux qui, faute d'en exploiter les avantages, en subissent les inconvénients, parfois terribles 5 .

Le don d'une photo ou d'une vidéo à une personne unique consiste en une confrontation à un autre réel, ce qui implique un risque nécessaire et incontournable lorsque l'individu tente de s'inscrire dans le lien social. Il s'agit de se « donner", de se risquer, mais aussi de vérifier l'effectivité de sa propre capacité personnelle à "s'assumer ", en se révélant " authentiquement » à l'interlocuteur désigné. Certains se dévoilent alors pour demander implicitement à l'autre ce qui relève de l'acceptable ou de l'inacceptable, voire du privé ou de l'intime (Hirdman 2010, Jauréguiberry 2011). Une photo sexy est ainsi un outil pour montrer quelque chose de soi, un médiateur pour s'abandonner puis renforcer un lien de confiance, mais aussi afin de poser une question à l'autre : cela relève-t-il seulement de notre intimité ? Au risque de la diffusion, le fait de garder pour soi, puis pour eux le document compromettant, participe d'une expérience de délimitation fragile, mais d'une délimitation tout de même de l'intimité. En conservant ce même document pour eux, les personnes signifient aussi, de manière implicite, que son contenu n'est pas socialement partageable puisqu'il n'est pas socialement acceptable. Un système d'auto-régulation prend ainsi forme (Sveningsson Elm 2009, Hirdman 2010, Schwarz 2010b), les frontières de l'intimité ne cherchant pas à être abolies, mais bien à se reconstruire dans un monde où elles ne sont pas toujours clairement identifiables.

\section{Se présenter sur les réseaux sociaux : se confronter au tribunal de l'image}

13 Si l'exposition de soi à une personne significative est l'étape qui succède souvent à la production de photo ou de vidéo pour soi, elle constitue aussi une étape avant que l'adolescent ne s'expose à un groupe plus large, dont il maîtriserait moins la qualité des éventuels commentaires. Le troisième cercle d'interlocuteurs implique donc un groupe, plus ou moins restreint, sur les réseaux sociaux. Cette fois, l'adolescent s'adresse à plusieurs personnes au même moment. Le désir d'extimité peut s'exprimer également ici : il s'agit de recevoir des retours pour s'ajuster, au besoin. Il est aussi possible de parler de confiance puisque l'exposition implique le risque de commentaires négatifs mais aussi de commentaires positifs renforçant éventuellement le sentiment d'appartenance. Toutefois, dans ce contexte des réseaux sociaux, la prise de risque apparaît dans une version plus 
radicale, puisque le silence de certains témoins ne signifie pas dans tous les cas leur approbation. En d'autres termes, la production de photos à destination des réseaux sociaux implique l'apprentissage parfois difficile, non seulement de la réception d'éventuelles désapprobations, mais également de l'incertitude entourant la perception que les autres ont de l'individu. Les performances se déploient alors, non seulement dans la confrontation au regard d'un groupe, mais aussi à travers sa capacité à résister, à intégrer, à départager, en un mot à choisir, ce qu'il conviendra de prendre et de laisser. Il s'agit moins d'un abandon d'une image de soi puis d'une réception de commentaire à sens unique que de l'entrée sur une scène où l'adolescent se risque au sein d'une interaction qu'il cherche à provoquer, à l'occasion sans résultat.

Les réseaux sociaux sont parfois comparables à un tribunal de l'image. Ce qui est jugé ici, c'est l'affirmation d'un style, une manière de se présenter aux autres, de montrer son corps, de le déguiser, de le modifier, etc. En d'autres termes, c'est une démarche de présentation de soi intimement liée à une démarche de construction de son identité qu'il s'agit de présenter devant ses éventuels juges. Comme au tribunal, celui dont on atteste ou non la crédibilité prend la parole. Il s'expose et s'affirme. La salle d'audience est ouverte, nombreux sont ceux à pouvoir y assister. Mais peu joueront le rôle des jurés, en s'impliquant dans la «cause » présentée, en émettant un jugement. Certains iront plus loin, ils plaideront pour ou contre, à la manière d'avocats. Sur les réseaux sociaux, nous assistons ainsi à l'exposition rituelle de «causes » consistant à défendre une direction prise dans le contexte d'un travail sur l'identité.

15 À l'analyse de sens commun qui consiste à dire qu'Internet ne participe qu'au décloisonnement de l'intimité et à l'explosion de l'exhibitionnisme s'oppose l'analyse de chercheurs qui confirment la production située d'interactions d'où émergent de nombreux interdits et des conventions de politesse fortement ritualisées (Balleys et Coll 2015). L'éthos de l'adolescent se déploie à la convergence d'une production personnelle d'une théâtralisation du vrai rencontrant dans les mondes numériques des limites exprimées par les interlocuteurs. En comparaison avec la photo ou la vidéo destinée à une seule et unique personne, tout se passe comme si les documents visuels destinés à un groupe plus ou moins restreint sur les réseaux sociaux impliquaient moins une demande de retour que la provocation d'un échange, sous le regard d'un groupe témoin toujours prêt à s'impliquer davantage ${ }^{6}$. Par ailleurs, si le partage intime d'une photo à une personne choisie implique un laisser-aller, une sorte d'abandon d'un code de présentation de soi au profit d'un portrait plus "authentique", la diffusion sur les réseaux sociaux demande plutôt une grande maitrise des codes de présentation de soi.

Dans le contexte des réseaux sociaux, l'éthos de l'adolescent implique d'être prêt à la prise de parole, voire à l'argumentation. Les murs des réseaux sociaux comme Facebook comptent parmi les espaces numériques les plus prisés: espaces d'échanges publics, ils sont investis comme des arènes où certains individus exposent leurs mises en scène, mais aussi où ils défendent le sens et la validité de ces dernières. Leurs performances se déploient alors en intégrant la dimension incontournable de la parole, du talent à rallier ou non les autres à leur cause. Lorsqu'il s'adresse à ce cercle d'interlocuteurs, l'adolescent passe de la mise en scène à la mise en mots. Il n'est plus seulement dans la découverte de lui-même, ni même dans la recherche de validation, mais aussi dans l'affirmation de soi qui ne peut jamais faire l'économie de la limite imposée par l'autre. Bien que fortement critiqués, notamment dans les médias, les réseaux sociaux favorisent chez la grande majorité des adolescents la convergence du paraître et de l'être (Jauréguiberry 2011). En 
réalité, les mondes numériques facilitent la convergence d'une démarche individuelle de construction de soi avec une confrontation socialisante avec le groupe. Les phénomènes nombreux, témoignant de la souffrance d'adolescents et d'adolescentes victimes de la violence verbale sur les réseaux sociaux, rendent visibles, non seulement la violence de certains interlocuteurs, mais aussi le difficile passage pour des ados de l'affirmation de soi par l'image à l'affirmation de soi par le discours.

Bien que l'image joue aujourd'hui un rôle prépondérant dans une démarche de construction de soi, l'éthos de l'adolescent à l'ère du numérique intègre aussi l'art de manier la parole. Celui qui présente une photo de lui-même doit être prêt à en défendre le contenu. En général, la défense d'un point de vue, d'une affirmation, d'un choix sur les réseaux sociaux mobilise les deux significations généralement attribuées à l'éloquence. D'une part, il s'agit effectivement de savoir persuader et séduire, de savoir convaincre en maniant le verbe, à travers une écriture qui respecte les conventions de la syntaxe et du vocabulaire «SMS ». D'autre part, il s'agit de mobiliser une éloquence faite de silence, d'absence, d'émoticône, avec l'ambiguïté que ce langage favorise. Les performances de l'adolescent dans les espaces numériques se parent tôt ou tard de la présence de mots qui fortifient, expliquent, justifient, convainquent les autres de la valeur des choix exposés sur Internet, que ce soit sur le registre des vêtements, des goûts musicaux ou cinématographiques, de ses préférences relationnelles, voire sexuelles. Autour des photos et vidéos qui provoquent des interactions se déploient des prises de parole et des échanges producteurs de sens, qui favorisent aussi la production d'un discours réflexif sur soi. En d'autres termes, au risque de l'image exposée s'ajoute le risque de la prise de parole en public. Par exemple, lorsqu'un adolescent présente une nouvelle photo de profil sur Facebook en affichant un nouveau style personnel, le risque inhérent à la mise en scène de soi implique qu'il devra peut-être répondre à certaines désapprobations. À une critique exprimée par un «ami » Facebook, il devra éventuellement proposer une réplique pour se défendre, d'où l'importance, non seulement de savoir se "montrer " mais aussi de savoir « argumenter».

\section{Se présenter sur YouTube : s'abandonner au regard anonyme des internautes}

Le quatrième cercle d'interlocuteurs se distingue des précédents par la dissimulation de leur identité. Il s'agit cette fois de s'adresser à un groupe indéfini dont on ne connait pas l'identité de chaque membre. Si sur les réseaux sociaux les échanges ne peuvent s'effectuer autrement qu'à travers une certaine réciprocité, sur YouTube, l'image est le plus souvent abandonnée au regard anonyme de la communauté des internautes. En ce sens, l'échange réciproque n'est pas recherché puisqu'extrêmement limité, pour ne pas dire impossible: en dessous des vidéos mises en ligne sur YouTube, des listes de commentaires s'allongent, se multiplient sans révéler l'identité de leurs émetteurs. Ici, à travers la mise en ligne d'images de soi, l'individu ne cherche pas à provoquer l'échange. La publication ne sert pas la production d'un discours sur soi et sur ses choix. Elle se résume plutôt à l'abandon d'une image de soi au regard de l'autre qui commente, au risque de la désapprobation radicale, ou qui reste muet, au risque de l'indétermination inhérente au silence. 
19 Dans ce contexte, le choix de révéler ou non son identité apparaît comme particulièrement significatif. En ne la dévoilant pas, tout en s'exposant dans une mise en scène, le producteur et diffuseur de la vidéo conserve avec ses locuteurs une certaine égalité : ni l'un ni l'autre ne sont identifiables. En revanche, lorsqu'il dévoile son identité, en montrant clairement son visage, ce producteur-diffuseur accepte délibérément d'entrer dans un rapport profondément inégalitaire, la réciprocité ne s'appliquant pas entre les interlocuteurs. Car celui qui est vu est connu, celui qui regarde ne l'est pas. Dans ce dernier cas, la prise de risque reprend une place importante au détriment d'une démarche de connaissance de soi que nous pourrions qualifier de plus prudente, non pas parce qu'elle évacue toutes formes de prises de risque, mais plutôt parce qu'elle implique une confrontation progressive à soi, à l'autre puis à l'inconnu. De plus, dans ce cas, l'image ne sert plus le basculement progressif de l'adolescent de l'ordre de l'image à celui de la parole. Au contraire, elle suppose un usage qui prend le corps comme objet pour se mettre en danger, bien que ce dernier ne soit pas mis physiquement à l'épreuve.

L'exemple des strip-teases en ligne illustre bien le contexte spécifique d'intelligibilité d'images diffusées auprès d'un groupe anonyme. Depuis l'avènement de YouTube en 2004, de nombreux strip-teases ont été publiés en ligne par des jeunes femmes. Si certaines révèlent frontalement leur identité, plusieurs optent pour un cadrage dissimulant scrupuleusement leur visage. Seul le corps qui se dénude est exposé aux yeux des internautes. Dans les deux cas, il s'agit sans surprise de susciter les commentaires, de trouver une certaine approbation auprès d'inconnus en un temps d'appropriation de son corps de femme. Mais ces deux cas renvoient dans les faits à deux figures distinctes du risque bien connues des anthropologues: les conduites ordaliques et les conduites sacrificielles (Le Breton 2002).

21 La socio-anthropologie de l'adolescence insiste depuis plusieurs années sur deux figures du risque fréquemment mobilisées par les ados. D'une part, le sacrifice consiste à abandonner une partie de soi pour sauver le tout. À l'échelle individuelle, il s'agit de sacrifier une part de soi. D'autre part, l'ordalie consiste plutôt à jouer le tout par le tout. Il s'agit de s'en remettre au hasard pour connaître l'issue d'une situation dangereuse, et de s'y abandonner délibérément. Depuis une dizaine d'années, les conduites sacrificielles et ordaliques observables dans les espaces physiques, mettant frontalement le corps en jeu, se sont déployées dans les espaces numériques, mettant plutôt l'image du corps en jeu, faisant ainsi glisser l'individu du risque de se blesser ou de mourir au risque de l'insulte et de la réputation bafouée (Lachance 2015). L'exemple des strip-teases en ligne mobilise souvent la logique du sacrifice et de l'ordalie. Il s'agit de montrer son corps en mouvement dans un moment fixé dans le temps, d'abandonner une partie rendue visible de son histoire, d'en sacrifier l'histoire cachée. Il s'agit aussi de s'en remettre au regard anonyme des internautes, en se confrontant au risque du commentaire tout aussi anonyme.

\section{Du glissement du statut à l'identité}

Traversant et mobilisant différents interlocuteurs et différents mondes numériques, l'éthos de l'adolescent se déploie au gré d'une démarche de construction de soi. La mobilisation importante des technologies de l'image et de la communication par les ados de tous les pays occidentaux ne saurait d'ailleurs s'expliquer simplement par l'accessibilité récente des mondes numériques. Nous pourrions imaginer un monde 
différent, dans lequel, par exemple, le refus des technologies aurait symbolisé la scission entre les générations. Nous pouvons également imaginer un usage, des appropriations et des détournements tout à fait différents dans une société où nos adolescents n'auraient pas été hypermodernes. Car l'investissement d'internet aujourd'hui exprime, en lui donnant une nouvelle visibilité, le travail singulier auquel nombre d'adolescents sont aujourd'hui confrontés lorsqu'il s'agit, simplement, de gagner en reconnaissance.

Destinée à lui-même, à une personne significative, à un groupe connu ou à un groupe anonyme, la production de photos et de vidéos est le plus souvent l'expression d'une quête de reconnaissance, impliquant une prise de risque par la confrontation au regard de l'autre, avec des intensités différentes selon l'espace investi. Si nous pouvons questionner l'étanchéité des frontières effectives entre ces quatre groupes, il est fort à parier que dans les représentations des jeunes internautes, c'est tantôt l'un tantôt l'autre que l'on croit atteindre. Dans tous les cas, l'éthos décrit ici souligne que les adolescents ne sont plus dans une quête de reconnaissance statutaire, mais bien de reconnaissance identitaire. Il ne s'agit pas d'être considéré comme des adultes, mais bien d'être reconnus comme des individus autonomes. Par conséquent, le regard de l'autre est recherché non pas afin que ce dernier assigne un statut, mais bien pour qu'il confirme la valeur d'une démarche que l'individu rend visible en exposant ses choix. Si l'éthos de l'adolescent à l'ère du numérique ne peut être totalement dissocié de celui des adultes, il se caractérise néanmoins par l'apprentissage progressif d'une confrontation au regard de l'autre, d'où des variations complexes, des interpellations de regards diversifiés, des risques pris, trahissant la nécessité d'avancer sur le chemin de l'autonomisation.

\section{BIBLIOGRAPHIE}

Balleys, Claire et Coll, Sami, 2015, « La mise en scène de la vie privée en ligne par les adolescents ", Reset, n 4, p. 2-17, [En ligne], http://reset.revues.org/547.

Goffman, Erving, 1973, La Mise en scène de la vie quotidienne. 1. La présentation de soi, Paris, Minuit.

Goguel d'Allondans, Thierry et Lachance, Jocelyn, 2014, Étudier les ados. Initiation à l'approche socioanthropologique, Rennes, Presses de l'EHESP.

Hirdman, Anja, 2010, « Vision and Intimacy: Gendered Communication Online », Nordicom Review, vol. $31, \mathrm{n}^{\circ} 1, \mathrm{p} .3-13$.

Jauréguiberry, Francis, 2011, « L'exposition de soi sur Internet :

un souci d'être au-delà du paraître ", dans N. Aubert et C. Haroche (dir.), Les Tyrannies de la visibilité. Le visible et l'invisible dans nos sociétés contemporaines, Ramonville-Saint-Agne, Érès, p. 131-145.

Jeffrey, Denis, Lachance, Jocelyn et Le Breton, David, 2016, Penser l'adolescence. Approche socioanthropologique, Paris, PUF.

Lachance, Jocelyn, 2011, L'Adolescence hypermoderne. Le nouveau rapport au temps des jeunes, Québec, Presses de l'Université Laval. 
Lachance, Jocelyn, 2012, Socio-anthropologie de l'adolescence, Québec, Presses de l'Université Laval, coll. « Lectures ».

Lachance, Jocelyn, 2013a, « Usages sociaux de la caméra numérique chez les jeunes. Autonomisation, interactions et identité », Agora débats/jeunesses, n 63, p. 37-51.

Lachance, Jocelyn, 2013b, Photos d'ados à l'ère du numérique, Québec, Paris, Presses de l'Université Laval, Hermann, coll. « Adologiques ».

Lachance, Jocelyn, 2015, « L'ordalie numérique », Adolescence, nº 33, p. 649-654.

Lavoie, Francine, 2013, Dénudé/e sur Internet : la nouvelle lettre d'amour ? L'usage de sextos chez les 14-25 ans, École de psychologie, Université Laval, Colloque interdisciplinaire de l'Université féministe d'été, [En ligne], http://adverbum.hautetfort.com/media/00/00/1274674074.pdf.

Le Breton, David, 2002, Conduites à risque, Paris, PUF.

Le Breton, David, 2008, Anthropologie du corps et modernité, Paris, PUF, coll. « Quadrige Essais Débats ».

Lenhart, Amanda, 2009, Teens and Sexting. How and Why Minor Teens are sending Sexually Suggestive Nude or Nearly Nude Images via Text Messaging, rapport de recherche, [En ligne], http:// www.pewinternet.org/files/old-media//Files/Reports/2009/PIP_Teens_and_Sexting.pdf.

Schwarz, Orit, 2010a, « Going to Bed with a Camera: on the Visualization of Sexuality and the Production of Knowledge », International Journal of Cultural Studies, vol. 13, nº 6, p. 637-656.

Schwarz, Orit, 2010b « Negotiating Romance in Front of the Lens », Visual communication, vol. 9, ${ }^{\circ}$ 2, p. 151-169.

Sveningsson Elm, Malin, 2009, « Teenagers get Undressed on the Internet. Young People's Exposure of Bodies in a Swedish Internet Community », Nordicom Review, vol. 30, n 2, p. 87-103.

Tisseron, Serge, 2010, « Extime », dans D. Le Breton et D. Marcelli (dir.), Dictionnaire de l'adolescence et de la jeunesse, Paris, PUF, p. 309-311.

\section{NOTES}

1. Comparativement au réseau social Facebook qui permet la publication de photos, vidéos et de commentaires au public élargi des « amis », Snapchat est une application smartphone facilitant la prise de photos rapidement, photos qu'il est possible de transmettre à une ou des personnes choisies. En tant que site de partage de vidéo en ligne, la plate-forme Youtube favorise quant à elle la diffusion de vidéo au plus grand nombre, tandis que Younow laisse aux internautes le loisir de regarder en direct les conversations d'autres internautes.

2. D'ailleurs, cette fortification de la confiance en soi se construit également lors de la production de photo. Dans le cadre d'une recherche qualitative sur la pratique du selfie, certains adolescents ont révélé que, dans le moment de la prise de photo, l'acte photographique était parfois vécu comme une forme d'affirmation de soi. En d'autres termes, se prendre soi-même en photo sous le regard des autres est une manière de signifier le fait que l'adolescent se trouve suffisamment beau ou belle pour se prendre effectivement comme objet d'une photo.

3. Snapchat est une application smartphone qui permet de prendre et de transmettre une photo pour une durée limitée, celle-ci disparaissant après un nombre de secondes déterminées par le transmetteur. 
4. Le clin d'œil à E. Goffman rappelle ici que les problématiques liées à la présentation de soi, à la «façade", à la peur de perdre la face, etc., sont antérieures à l'avènement des espaces numériques.

5. Nous pensons notamment aux victimes de cyberharcèlement.

6. Claire Balleys remarque à cet effet que la quantité d'amis Facebook n'exclut pas la recherche de relations intimes, entretenues en ligne. Certains adolescents vont ainsi utiliser leur mur afin de laisser paraître des relations exclusives au plus grand nombre avant de poursuivre l'échange en message privé.

\section{RÉSUMÉS}

À partir d'une lecture socio-anthropologique, cet article propose d'analyser les performances corporelles des adolescents dans les mondes numériques, en insistant sur la place occupée par le destinataire des photos et des vidéos produites. En distinguant à des fins d'analyse les documents visuels produits pour soi, partagés avec Snapchat, Facebook ou YouTube, ces performances prendraient alors leur sens dans des interactions situées qui nécessitent la connaissance du destinataire pour être analysé d'où la constitution d'un éthos numérique singulier à l'adolescence.

From a socio-anthropological point of view, this article aims to analyze corporal performances by teenagers in digital worlds, emphasizing the place of the recipient of produced photos and videos. For the purposes of analysis, thoses visuals documents are seing throught four kinds of interlocutors: the document produced for itself, shared with Snapchat, Facebook or YouTube. These performances would take their meaning in situated interactions that require the identity of the recipient to be entered, then appears a specific digital ethos during the adolescence.

\section{INDEX}

Mots-clés : adolescents, réseaux sociaux, images, photos, performances, corps, Snapchat, Facebook, YouTube

Keywords : teenagers, social network, body

\section{AUTEUR}

\section{JOCELYN LACHANCE}

Université de Pau et des Pays de l'Adour 